\title{
EFEITOS DO RESFRIAMENTO E AQUECIMENTO ARTICULAR NO DESEMPENHO FUNCIONAL DO OMBRO
}

\author{
EFFECTS OF COOLING AND HEATING ON SHOULDER FUNCTIONAL PERFORMANCE
}

Artigo Original

ORIGINAL ARTICLE

EFECTOS DEL ENFRIAMIENTO Y DEL CALENTAMIENTO ARTICULAR DEL HOMBRO

Artículo Original

EN EL DESEMPEÑO FUNCIONAL

Dayane de Oliveira Estevam (Graduanda em Fisioterapia)

Marcelo Lima de Oliveira ${ }^{1}$

(Graduando em Fisioterapia)

Marcelo Lourenço da Silva'

(Fisioterapeuta)

Leonardo César Carvalho

(Fisioterapeuta)

Daniel Ferreira Moreira Lobato

(Fisioterapeuta)

1. Universidade Federal de Alfenas Alfenas, MG, Brasil.

\section{Correspondência:}

Daniel Ferreira Moreira Lobato Universidade Federal de Alfenas Unidade Educacional II.

Av. Jovino Fernandes Salles, 2600.

Santa Clara. Alfenas, MG, Brasil.

37130-000.

daniellobato@ig.com.br

\section{RESUMO}

Introdução: Um dos recursos mais utilizados na reabilitação de lesões musculoesqueléticas é a termoterapia por subtração de calor (crioterapia), enquanto a termoterapia por adição de calor é considerada o procedimento mais antigo de reabilitação física. Entretanto, há poucas evidências que tenham investigado os efeitos desses recursos sobre o desempenho de membros superiores. Objetivo: Comparar o efeito do resfriamento e do aquecimento articular sobre o desempenho funcional do membro superior. Métodos: Trinta e quatro voluntários $\left(22,23 \pm 2,17\right.$ anos; $\left.22,39 \pm 2,53 \mathrm{~kg} / \mathrm{m}^{2}\right)$, de ambos os sexos, foram divididos aleatoriamente em um dos três grupos: 1) grupo crioterapia $\mathrm{GCR}(\mathrm{n}=10)$ : submetidos ao resfriamento articular por compressas frias; 2 ) grupo termoterapia - GTE ( $n=10)$ : submetidos ao aquecimento articular por ondas curtas e 3) grupo controle - GCO ( $n=14)$, não submetidos a qualquer intervenção. Os voluntários foram avaliados, pré e pós-intervenção, quanto ao desempenho funcional de membros superiores por meio dos testes de estabilidade da extremidade superior em cadeia cinética fechada (TEESCCF) e das condições de equilíbrio em apoio bimanual sobre o baropodômetro. Ainda, os voluntários foram avaliados quanto ao desempenho funcional virtual por meio do jogo Mario Kart (Nintendo Wii ${ }^{\circledR}$ ). Resultados: Houve melhora significativa nos valores pós-intervenção no TEESCCF para o GCR $(p<0,001)$, GTE $(p=0,002)$ e GCO $(p=0,01)$. Não houve alteração significativa na área de deslocamento do centro de pressão na condição de olhos abertos, nos três grupos ( $p>0,05)$. Entretanto, na condição de olhos fechados, houve piora de desempenho para GTE $(p=0,04)$ e melhora de desempenho para o $\mathrm{GCO}(\mathrm{p}=0,02)$. Não houve alteração significativa no desempenho funcional virtual para os três grupos $(p>0,05)$. Conclusão: Embora não tenha favorecido o desempenho funcional em todos os testes reais e virtuais utilizados, o resfriamento articular foi mais efetivo que o aquecimento articular para manter o desempenho muscular do membro superior, especialmente nas condições de equilíbrio sobre membros superiores na condição de olhos fechados.

Palavras-chave: diatermia, agentes de resfriamento, equilíbrio postural, extremidade superior.

\section{ABSTRACT}

Introduction: Thermotherapy by heat subtraction or cryotherapy is one of the most used resources in the physical rehabilitation whereas thermotherapy by addition of heat is considered as the oldest procedure in physical rehabilitation. However, there are few evidences regarding the effects of these physical agents on the performance of upper limbs. Objective: To compare the effect of joint cooling and joint heating on functional performance of the upper limb. Methods: Thirty-four subjects $\left(22.23 \pm 2.17\right.$ years old; $\left.22.39 \pm 2.53 \mathrm{~kg} / \mathrm{m}^{2}\right)$ of both genders were randomly assigned to one of the following three groups: 1) subjects who were submitted to joint cooling with cold compresses - CRG $(n=10) ; 2)$ subjects who were submitted to joint heating by shortwave thermotherapy - THG $(n=10)$ and 3) subjects who were not submitted to any intervention - control group - COG ( $n=144$ ). Upper limb performance was assessed pre-and post-intervention by the closed kinetic chain upper extremity stability test (CKCUEST). Balance conditions were assessed in upper limb supporting on a pressure platform. Virtual performance was evaluated by Mario Kart Wii game (Nintendo Wi ${ }^{\circledR}{ }^{\circledR}$. Results: There was significant improvement in post intervention measurements in CKCUEST for both CRG $(p<0.001)$, THG $(p=0.002)$ and COG $(p=0.01)$. There were no significant changes in the center of pressure area displacement with opened eyes in the three groups ( $p>0.05)$. However, in the closed eyes condition, there was a worsened performance for $T H G(p=0.04)$ and improved performance for $C O G(p=0.02)$. No significant changes in virtual performance were observed for the three groups $(p>0.05)$. Conclusion: Joint cooling was more effective than joint heating to maintain the upper limb muscle performance, especially in upper limb balance with closed eyes although it did not favor functional performance in all real and virtual tests.

Keywords: diathermy, cooling agents, postural balance, upper extremity.

\section{RESUMEN}

Introducción: Uno de los recursos más utilizados en la rehabilitación de lesiones musculoesqueléticas es la termoterapia por sustracción de calor (crioterapia), mientras que la termoterapia por agregado de calor es considerada como el procedimiento más antiguo de la rehabilitación física. Entretanto, hay pocas evidencias de que se hayan investigado los efectos de esos recursos sobre el desempeño de miembros superiores. Objetivo: Comparar el efecto del enfriamiento y 
del calentamiento articular sobre el desempeño funcional del miembro superior. Métodos: Treinta y cuatro voluntarios $\left(22,23 \pm 2,17\right.$ años; $\left.22,39 \pm 2,53 \mathrm{~kg} / \mathrm{m}^{2}\right)$, de ambos sexos, fueron divididos randómicamente a uno de los tres grupos: 1) grupo crioterapia GCR ( $n=10)$ sometidos al enfriamiento articular por compresas frías; 2$)$ grupo termoterapia - GTE ( $n$ = 10) sometidos al calentamiento articular por ondas cortas y 3) grupo control - $G C O(n=14)$, no sometidos a ninguna intervención. Los voluntarios fueron evaluados antes y después de la intervención, cuanto al desempeño funcional de los miembros superiores a través de los tests de estabilidad de la extremidad superior en cadena cinética cerrada (TEESCCF) y de las condiciones de equilibrio en apoyo bimanual en un baropodómetro. Además, los voluntarios fueron evaluados cuanto al desempeño funcional virtual a través del juego Mario Kart (Nintendo Wi ${ }^{\circledR}$ ). Resultados: Hubo mejora significativa en los valores post-intervención en TEESCCF para GCR $(p<0,001)$, GTE $(p=0,002)$ y GCO $(p=0,01)$. No hubo alteración significativa en el área de desplazamiento del centro de presión en la condición de ojos abiertos, en los tres grupos $(p>0,05)$. Entretanto, en la condición de ojos cerrados, empeoró el desempeño de GTE $(p=0,04)$ y mejora en el desempeño funcional para el GCO ( $p=0,02)$. No hubo alteración significativa en el desempeño funcional virtual para los tres grupos $(p>0,05)$. Conclusión: A pesar de que no se haya favorecido el desempeño funcional en todos los tests reales y virtuales utilizados, el enfriamiento articular fue más eficaz que el calentamiento articular para mantener el desempeño muscular del miembro superior, especialmente en las condiciones de equilibrio sobre miembros superiores en la condición de ojos cerrados.

Palabras clave: diatermia, agentes de enfriamiento, equilibrio postural, extremidad superior.

\section{INTRODUÇÃO}

A termoterapia consiste na aplicação terapêutica de recursos físicos que promovem variação da temperatura tecidual, na forma de adição (termoterapia por adição) ou subtração (termoterapia por subtração ou crioterapia) de calor ${ }^{1}$. A termoterapia por subtração de calor ou crioterapia consiste na aplicação de qualquer substância para remover o calor do corpo, resultando na diminuição da temperatura tecidual². Embora esteja bem fundamentada em relação a sua eficácia após lesões traumáticas, ainda há divergências quanto às alterações na função neuromuscular em decorrência do resfriamento tecidual promovido por esse recurso. Sugere-se que a crioterapia possa influenciar a atividade elétrica muscular, bem como os comandos periféricos responsáveis pelo ajuste dessa atividade, afetando negativamente a força muscular ${ }^{3}$, o que poderia tornar o atleta ou o individuo fisicamente ativo suscetível às lesões ${ }^{4}$. Contudo, diante dos achados de redução ${ }^{5}$, aumento ${ }^{6}$ e não-interferência ${ }^{7}$ do resfriamento tecidual sobre a força muscular, as dúvidas e divergências quanto ao desenvolvimento de atividades físicas imediatamente após o uso deste recurso permanecem.

A termoterapia por adição de calor é considerada o procedimento mais antigo que se tem conhecimento na prática de reabilitação físi$\mathrm{ca}^{8}$, apresentando benefícios relacionados ao aumento da temperatura muscular e do metabolismo energético, da função do sistema nervoso central e do recrutamento das unidades motoras ${ }^{9}$, resultando em maior velocidade de reação e coordenação dos movimentos ${ }^{10}$. Por essas razões, verifica-se que em atletas o aquecimento é uma prática amplamente utilizada para a melhora do desempenho esportivo, embora haja poucas evidências sobre os seus reais efeitos no desempenho muscular.

Neste sentido, reconhece-se que tanto o resfriamento quanto o aquecimento tecidual ainda não apresentam esclarecimento adequado a respeito de seus efeitos sobre o sistema neuromuscular, previamente a uma atividade esportiva. Além disso, os estudos que abordaram os efeitos dessas modalidades sobre a função do membro superior são escassos. O desempenho do membro superior é fundamental para diversas atividades esportivas e do contexto diário. Logo, reconhecer os efeitos de diferentes modalidades de temperatura sobre a função dos membros superiores parece fundamental para subsidiar o uso desses recursos e as suas eventuais contraindicações.

O desempenho funcional de membros superiores pode ser avaliado, de forma objetiva, por meio de diversos testes funcionais. Embora não usual, a estabilometria, que consiste na mensuração da distribuição da pressão e dos deslocamentos do centro de pressão durante a posição ortostática ou em movimento sobre uma plataforma de pressão, foi recentemente sugerida para avaliar indiretamente a estabilidade de membros superiores em cadeia cinética fechada ${ }^{11}$. Outro recurso de uso crescente na avaliação e intervenção fisioterapêutica é a realidade virtual, na qual os indivíduos podem executar tarefas com segurança e de forma completa, simulando situações reais ${ }^{12}$. Estima-se que o uso dessa tecnologia possa trazer avanços não apenas em protocolos de tratamento, mas também na avaliação funcional em situações associadas ou não a disfunções musculoesqueléticas.

Diante do exposto, verifica-se uma divergência de achados entre os estudos que avaliam os efeitos do resfriamento e do aquecimento tecidual sobre a força e sobre o desempenho muscular. Além disso, não foi verificado nenhum estudo que tenha investigado o desempenho funcional de membros superiores por meio da realidade virtual e por meio da estabilometria adaptada para avaliação de membros superiores. Deste modo, o objetivo deste estudo será avaliar, de forma comparativa, a influência do resfriamento e do aquecimento muscular local de ombro e braço no desempenho funcional de membros superiores de indivíduos saudáveis, em situações reais e virtuais.

\section{MÉTODOS}

O presente estudo consiste em um ensaio clínico, randomizado, transversal e quantitativo, aprovado pelo Comitê de Ética e Pesquisa da Universidade Federal de Alfenas (UNIFAL-MG) - parecer n 637.818/2014.

A amostra foi constituída por 34 indivíduos, sendo 26 do sexo feminino e oito do sexo masculino, com idade entre 18 e 30 anos, pertencentes à comunidade universitária. Todos assinaram um Termo de Consentimento Livre e Esclarecido, de acordo com a resolução 196/96 do Conselho Nacional de Saúde. Foram adotados como critérios de inclusão: presença de boas condições de saúde, bem como a participação regular em atividades físicas ${ }^{13}$. Foram considerados critérios de exclusão: a) história de cirurgia e/ou disfunção ortopédica nos membros superiores e coluna vertebral, b) presença de doença cardiovascular ou respiratória que impedisse a realização dos movimentos em estu$\mathrm{do}^{14}, \mathrm{c}$ ) relato de qualquer história de lesão pelo resfriamento ou pelo aquecimento tecidual, d) distúrbios de termorregulação e e) consumo de álcool nas últimas 24 horas antes da avaliação ${ }^{15}$.

A amostragem selecionada foi alocada aleatoriamente, por sorteio simples, em um dos três grupos: 1) - grupo crioterapia (GCR, $n=10)$ : 
sujeitos submetidos ao resfriamento articular de ombro e braço por compressas frias; 2) grupo termoterapia por adição de calor ( $G T E, n=10)$ : sujeitos submetidos ao aquecimento articular de ombro e braço por ondas curtas; e 3) grupo controle (GCO, n=14): sujeitos não submetidos a qualquer tipo de intervenção.

A coleta dos dados foi realizada no Laboratório de Análise do Movimento da UNIFAL-MG, na cidade de Alfenas-MG, Brasil. Inicialmente foi realizada uma entrevista e avaliação física para verificação dos critérios de inclusão e exclusão, bem como para registro de dados demográficos e antropométricos. Em seguida, foi avaliado o desempenho funcional real realizando-se o teste de estabilidade da extremidade superior em cadeia cinética fechada - TEESCCF. Para tanto, duas tiras de fitas adesivas foram fixadas ao solo, de forma paralela, com uma distância de $90 \mathrm{~cm}$ (sexo masculino) e $60 \mathrm{~cm}$ (sexo feminino) entre elas, conforme adaptação de Roush et al. ${ }^{16}$ Partindo de uma posição de apoio em flexão de braços, com cada uma das mãos sobre uma das fitas (ponto de referência=30 dedo) (figura 1), o sujeito foi instruído a tocar a mão oposta e voltar à posição inicial de apoio bimanual, repetindo o movimento com o membro superior oposto e alternando os movimentos durante o tempo de 15 segundos, da forma mais rápida possível. Os toques válidos (que sobrepusessem à mão contralateral, sobre a fita) foram contabilizados e representaram a medida de desempenho no teste. Após a devida familiarização dos sujeitos, foram realizadas três execuções do teste, com um período de descanso de 45 segundos entre cada execução, e utilizada a melhor medida de desempenho para posterior análise ${ }^{16}$.

Em seguida, foi realizada a avaliação das condições de equilíbrio em apoio bimanual por estabilometria no baropodômetro FootWork Pro (IST Informatique, França), com intuito de verificar a área de deslocamento do centro de pressão na condição de olhos abertos e fechados, como medida indireta de estabilidade de membro superior. $O$ centro de pressão consiste em uma medida de deslocamento indicativa da trajetória do vetor da força de reação do solo (FRS) resultante da plataforma de força, sendo correspondente a uma medida ponderada dos pontos de aplicação de todas as forças que atuam em sentido caudal na plataforma de força ${ }^{17}$. Para tanto, os indivíduos foram posicionados com os membros superiores em posição de flexão de braços, com cotovelos em semiflexão e com as mãos espalmadas sobre a área ativa (40 X $40 \mathrm{~cm}$ ) da plataforma do baropodômetro $(57,5 \mathrm{~cm}$ de comprimento $X 45 \mathrm{~cm}$ de largura $X 2,5 \mathrm{~cm}$ de altura) e os membros inferiores apoiados em uma bola suiça de 55 centímetros, para gerar maior instabilidade e desafio à tarefa (figura 2). 0 indivíduo foi orientado a manter a posição de apoio bimanual, o mais estável possível, durante 10 segundos. Após a devida familiarização dos sujeitos, o teste foi realizado em duas

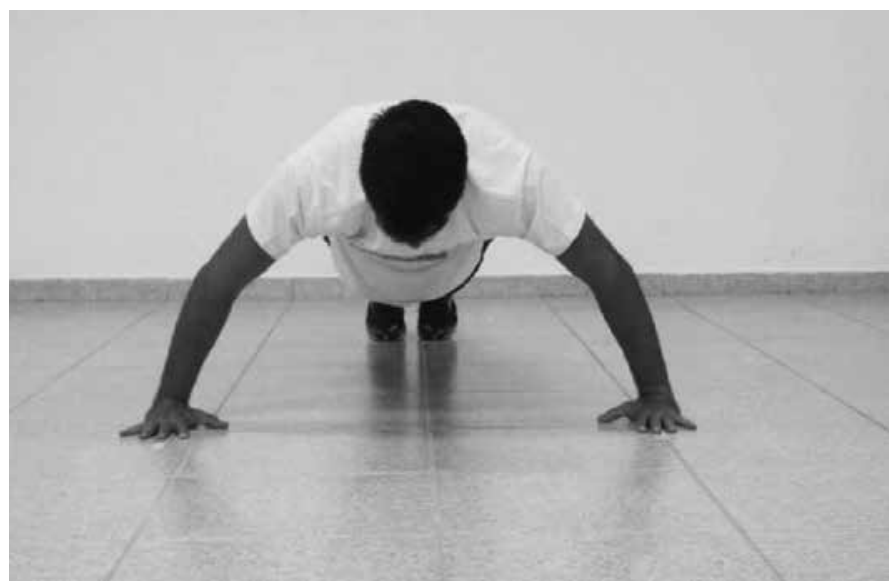

Figura 1. Posicionamento do voluntário para a realização do teste de estabilidade da extremidade superior em cadeia cinética fechada (TEESCCF). condições: com olhos abertos (uma vez) e com olhos fechados (uma vez), com repouso de um minuto entre um teste e outro, a fim de evitar os efeitos da fadiga muscular. Os dados foram adquiridos a uma freqüência de aquisição de $100 \mathrm{~Hz}$ e analisados no software FootWork Pro v. 3.2.2.0 (IST Informatique, França).

O desempenho funcional virtual de membros superiores foi avaliado por meio da utilização de um videogame Nintendo Wii ${ }^{\circledR}$, acompanhado do jogo Mario Kart, no qual o sujeito controla o kart virtualmente através do controle do videogame utilizando o acessório Wii Wheel ${ }^{\circledR}$, semelhante a um volante de automóvel, transmitindo a sensação de dirigir como no cotidiano. Este acessório permite um controle mais intuitivo e convencional no momento de jogar, especialmente no momento de realizar curvas. No presente estudo, o jogo foi realizado em pista livre, sem adversários, com o objetivo de atingir o menor tempo possível por volta, assim estabelecendo (ou não) a melhora do seu desempenho. Inicialmente houve uma familiarização do sujeito com o jogo, na qual foram realizadas nove voltas em um circuito pré-definido. Para tanto, o sujeito foi posicionado sentado em uma cadeira, de forma confortável, com o ombro em posição neutra e cotovelos mantidos a aproximadamente $90^{\circ}$ de flexão, segurando o acessório Wii Wheel ${ }^{\circledR}$ (figura 3). Em seguida à familiarização, foram realizadas nove voltas no mesmo circuito, para o registro do desempenho virtual. Foi registrado o tempo gasto para realizar cada volta e utilizado o tempo da melhor volta de cada individuo para posterior análise.

As avaliações de desempenho funcional foram realizadas antes da intervenção e imediatamente após a aplicação da mesma. Os indivíduos que compuseram o grupo controle permaneceram durante $30 \mathrm{mi}$ -

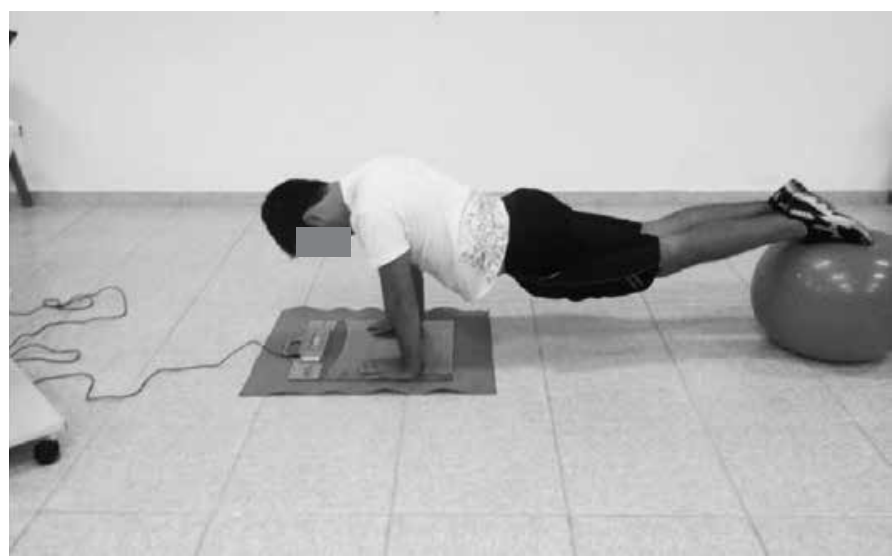

Figura 2. Posicionamento do voluntário para a avaliação das condições de equilíbrio em apoio bimanual sobre o baropodômetro.

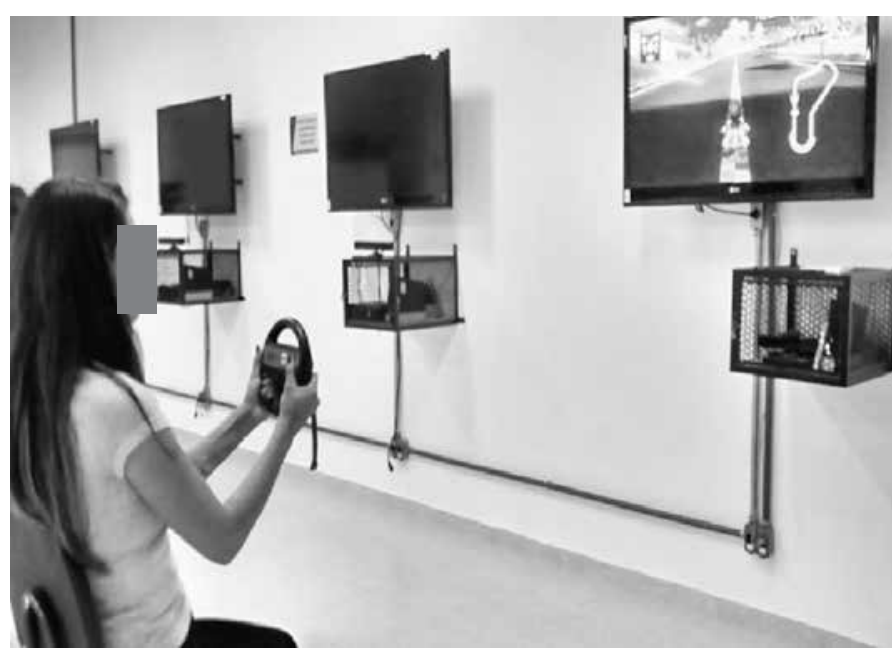

Figura 3. Posicionamento do sujeito para a realização do teste de desempenho virtual no jogo Mario Kart. 
nutos aguardando para que fosse realizada uma nova avaliação. Quanto às intervenções, o resfriamento articular por crioterapia foi realizado por meio da aplicação de compressas frias de gel $\left(\right.$ Mercur $\left.^{\circledR}\right)$ na articulação do ombro, bilateralmente, durante 20 minutos. A termoterapia por adição de calor foi realizada por meio da aplicação de diatermia por ondas curtas (Thermopulse ${ }^{\circledR}$ ), bilateralmente, durante 20 minutos, com os eletrodos aplicados de acordo com a técnica longitudinal, sendo um inferior à escápula (em decúbito dorsal) e outro na face anterior/ lateral do braço.

\section{Análise estatística}

A análise estatística foi realizada no programa STATISTICA for Windows - versão 5.0 (Statsoft Inc., Tulsa, OK, USA). A normalidade e a homocedasticidade dos dados foi verificada por meio dos testes de Kolmogorov-Smirnov e de Levenne, respectivamente. Para a análise dos dados demográficos e antropométricos, foi utilizada a análise de variância para um fator (GRUPO) - ANOVA ONE-WAY. Para a análise do efeito das intervenções intragrupo foi utilizado o teste $t$ para amostras dependentes, enquanto as comparações intergrupos foram avaliadas por meio de análise de variância para dois fatores (GRUPO e PERÍODO) - ANOVA TWO-WAY, com medidas repetidas. Quando necessário, conduziu-se o teste de comparação múltipla de Tukey, após as análises de variância. Em todas as situações foi utilizado um nível de significância de 5\%.

\section{RESULTADOS}

Em relação aos dados demográficos e antropométricos, houve efeito significativo apenas para a variável massa corporal $(p=0,04)$, com o GCR apresentando valores significativamente superiores ao GTE $(p=0,03)$ (tabela 1).

Em relação ao desempenho funcional virtual, não houve diferença significativa entre os valores da $1^{\mathrm{a}}$ e da $2^{\mathrm{a}}$ avaliações, para os três grupos. Do mesmo modo, quanto às comparações intergrupos, não houve efeito ou interação significativos para os fatores analisados ( $p>0,05)$.

Em relação ao desempenho funcional no TEESCCF, houve diferença significativa entre os valores da $1^{\text {a }}$ e da $2^{\mathrm{a}}$ avaliações para os três grupos, com melhores valores de desempenho na $2^{\mathrm{a}}$ avaliação. Quanto às comparações intergrupos, houve efeito significativo para os fatores GRUPO ( $p<0,001)$ e PERÍODO ( $p=0,003)$, sendo evidenciada diferença significativa entre o desempenho funcional inicial do GCR em relação: a) ao desempenho inicial do $G C O(p=0,005)$; b) ao desempenho final do GCO $(p<0,001)$ e $c)$ ao desempenho final do GTE $(p=0,001)$, com valores inferiores para o GCR (tabela 2).

Em relação ao desempenho funcional de membros superiores na estabilometria na condição de olhos abertos, não houve diferença significativa entre os valores da $1^{\text {a }}$ e da $2^{\mathrm{a}}$ avaliações, para os três grupos. Quanto às comparações intergrupos, houve efeito significativo para o fator GRUPO $(p<0,001)$, mas não para o fator PERÍODO $(p=0,64)$, sendo evidenciada diferença significativa entre o desempenho funcional inicial e final do GTE em relação a todos os demais grupos e condições (tabela 2). Na condição de olhos fechados, houve melhora significativa do desempenho para o $G C O(p=0,02)$ e piora significativa do desempenho para o GTE $(p=0,04)$, sem alteração de desempenho

Tabela 1. Média e desvio-padrão dos dados demográficos e antropométricos dos participantes.

\begin{tabular}{c|c|c|c|c}
\hline & GCR & GTE & GCO & p-valor \\
\hline Idade (anos) & $22,60 \pm 3,56$ & $22,30 \pm 1,25$ & $21,90 \pm 1,38$ & 0,76 \\
\hline Massa corporal $(\mathrm{kg})$ & $74,00 \pm 19,78$ & $59,00 \pm 11,00$ & $65,00 \pm 7,00$ & $0,04^{*}$ \\
\hline Altura $(\mathrm{m})$ & $1,72 \pm 0,11$ & $1,63 \pm 0,10$ & $1,69 \pm 0,08$ & 0,90 \\
\hline Índice de massa corporal $\left(\mathrm{kg} / \mathrm{m}^{2}\right)$ & $24,40 \pm 3,34$ & $22,00 \pm 2,00$ & $22,67 \pm 2,83$ & 0,14 \\
\hline
\end{tabular}

Tabela 2. Valores de média e desvio padrão pré e pós-intervenção nos grupos crioterapia (GCR), termoterapia (GTE) e controle (GCO), em relação ao teste de estabilidade da extremidade superior em cadeia cinética fechada (TEESCCF), desempenho virtual e área de deslocamento do centro de pressão com os olhos abertos (CP-OA) e fechados (CP-OF).

\begin{tabular}{c|c|c|c|c|c|c}
\hline \multirow{2}{*}{} & \multicolumn{2}{|c|}{ GCR } & \multicolumn{2}{c|}{ GTE } & \multicolumn{2}{c}{ GCO } \\
\cline { 2 - 7 } & Pré & Pós & Pré & Pós & Pré & Pós \\
\hline $\begin{array}{c}\text { TEESCCF } \\
\text { (toques) }\end{array}$ & $15,70 \pm 1,21$ & $19,30 \pm 3,77 *$ & $19,50 \pm 3,50$ & $22,20 \pm 3,93 *$ & $21,00 \pm 3,40$ & $22,57 \pm 3,29^{*}$ \\
\hline $\begin{array}{c}\text { Desempenho } \\
\text { virtual(s) }\end{array}$ & $31,56 \pm 5,30$ & $30,08 \pm 1,21$ & $30,82 \pm 0,91$ & $30,60 \pm 0,55$ & $30,79 \pm 1,26$ & $30,41 \pm 0,87$ \\
\hline CP-OA (cm $\left.{ }^{2}\right)$ & $1,10 \pm 1,31$ & $1,90 \pm 2,12$ & $5,66 \pm 2,34$ & $5,89 \pm 3,47$ & $2,47 \pm 2,37$ & $2,23 \pm 1,54$ \\
\hline CP-OF $\left(\mathrm{cm}^{2}\right)$ & $0,98 \pm 0,68$ & $0,95 \pm 0,60$ & $4,32 \pm 2,08$ & $6,53 \pm 3,08^{*}$ & $2,87 \pm 1,65$ & $2,15 \pm 1,63^{*}$ \\
\hline
\end{tabular}

para o GCR. Quanto às comparações intergrupos, houve efeito significativo para o fator GRUPO ( $p<0,001)$ e para a interação GRUPO $X$ PERÍODO $(p=0,02)$, mas não para o fator PERÍODO $(p=0,27)$, sendo evidenciada diferença significativa entre o desempenho funcional inicial do GTE em relação ao desempenho inicial e final do GCR, bem como do desempenho funcional final do GTE em relação a todos os demais grupos e condições (tabela 2).

\section{DISCUSSÃO}

Os resultados do presente estudo revelaram que na realização do TEESCCF houve melhora significativa de desempenho após a reavaliação, nos três grupos. Em relação ao GCR, esse resultado diverge de alguns estudos $^{16-18}$, que verificaram que o desempenho e a força muscular foram prejudicados pelo resfriamento local. Entretanto, nossos resultados corroboram indiretamente os achados de outro estudo ${ }^{6}$, que verificou aumento na produção de força muscular imediatamente após o resfriamento muscular. Outros resultados revelam que o resfriamento do tecido não altera a percepção da carga imposta ${ }^{19}$ ou a produção de força ${ }^{20}$, sugerindo que os métodos usuais de resfriamento (como o utilizado no presente estudo), não alteram o desempenho motor. Uma recente revisão sistemática verificou que a maioria dos estudos aponta diminuição da força muscular imediatamente após a aplicação do gelo, indicando que atletas de elite devem fazer curtas aplicações de gelo e/ ou realizar aquecimento à prática do esporte ${ }^{18}$. Entretanto, ressalta-se que esses dados são afirmados apenas para atletas de elite e, considerando a heterogeneidade de estudos, não trazem suporte adequado para a prática clínica, especialmente para indivíduos de menor aptidão física.

Neste sentido, uma possível explicação para os resultados encontrados nos três grupos pode ocorrer em função de um maior processo de adaptação dos participantes em relação ao teste, ainda que cuidados tenham sido realizados no sentido de permitir a adequada familiarização dos sujeitos em relação a cada etapa das avaliações. Contudo, observa-se que o grupo submetido ao resfriamento articular apresentou, do ponto de vista clínico, melhora evidente do desempenho funcional após a intervenção, quando comparado com os demais grupos, o que não descarta a hipótese de que os efeitos do resfriamento podem até mesmo favorecer o desempenho muscular ${ }^{6}$. Considerando que a crioterapia diminui a velocidade de condução nervosa ${ }^{2}$, alterando a resposta aferente do local submetido ao resfriamento e refletindo na diminuição da dor e da resposta de fadiga muscular ${ }^{21}$, supomos que o participante possa ter realizado a tarefa com uma menor percepção de esforço, apresentando maior empenho a esta tarefa e favorecendo, por conseqüência, seu desempenho, apesar de este fato representar aumento do risco de lesão por sobrecarga muscular.

Em relação à elevação da temperatura muscular, nossos resultados evidenciam que houve uma melhora significativa no desempenho funcional apenas no TEESCCF. A elevação da temperatura muscular 
aumenta a taxa de atividade da ATPase, o que pode promover a ativação mais rápida da fibra muscular ${ }^{22}$, em função do aumento da velocidade de condução do impulso nervoso, resultando em maior velocidade de reação e coordenação dos movimentos ${ }^{23}$. Entretanto, considerando que o grupo controle também apresentou uma melhora significativa no seu desempenho neste teste, sugere-se um importante papel do processo de aprendizado do participante em relação ao teste funcional e que o aquecimento local talvez não tenha sido o fator mais importante para justificar os ganhos de desempenho no GTE. Neste sentido, nossos resultados colocam em dúvida a afirmação de que o aquecimento leva necessariamente a uma melhora do desempenho muscular, visto que o único grupo a apresentar piora do desempenho funcional no teste de equilíbrio em apoio bimanual foi o submetido à termoterapia por adição de calor. Deste modo, é possível que o aquecimento tecidual não represente melhora sobre todos os aspectos funcionais do membro superior, o que requer investigações futuras.

Ainda que não existam estudos que tenham avaliado o desempenho muscular por meio da realidade virtual, ressaltamos que os achados deste estudo não revelaram alteração significativa de desempenho nos diferentes grupos. Neste sentido, consideramos que o jogo utilizado tenha gerado baixa demanda sobre a função do ombro, envolvendo contribuições mais importantes das articulações distais do membro superior. Deste modo, o uso de jogos que desafiam em maior intensidade a habilidade, a coordenação, as estratégias de reação e a força muscular do membro superior, em especial do complexo articular do ombro, é desejável e deve ser estimulado em pesquisas futuras.

Em relação à estabilometria, ressaltamos igualmente a existência de poucos estudos para efeito de comparação, o que dificulta a análise de nossos resultados. Destacam-se achados prévios ${ }^{11}$ que confirmaram a confiabilidade do uso da plataforma de força para a avaliação da estabilidade de membro superior ${ }^{11}$, subsidiando o seu uso no presente estudo, apesar das diferenças metodológicas. Na realização do teste com os olhos abertos, não foi obtida alteração significativa de desempenho em relação aos grupos. Entretanto, ao analisar o deslocamento do centro de pressão com os olhos fechados, que exige maior controle cinestésico e neuromotor, houve melhora significativa de desempenho no grupo controle, sugerindo também uma possível adaptação dos participantes deste grupo com o teste. O resfriamento articular não repercutiu em variação de desempenho da análise do centro de pressão com os olhos fechados, enquanto o aquecimento articular repercutiu em uma redução de desempenho, demonstrando maior instabilidade sobre os membros superiores durante a tarefa após a aplicação do recurso, talvez influenciado por um processo de fadiga muscular.

Neste sentido, consideramos como uma possível limitação do estudo a indução de um processo de fadiga muscular de forma diferenciada entre os diferentes grupos, apesar dos cuidados apontados em relação à alocação da amostra e familiarização dos sujeitos. Destacamos também a necessidade de realização de pesquisas com um maior número de indivíduos, para que os resultados possam ser mais representativos. Ainda, aponta-se a escassez de estudos utilizando a estabilometria adaptada para o membro superior e das medidas de desempenho virtual para avaliação da funcionalidade de membros superiores, o que requer a elaboração de mais estudos consistentes e de rigor metodológico, para que essas técnicas possam ter repercussão favorável à prática clínica.

\section{CONCLUSÃO}

Embora não tenha favorecido o desempenho funcional em todos os testes utilizados, o resfriamento articular foi mais efetivo que o aquecimento articular para manter o desempenho muscular do membro superior, especialmente nas condições de equilíbrio sobre membros superiores na condição de olhos fechados.

\section{AGRADECIMENTOS}

Os autores agradecem ao Apoio Financeiro da Fundação de Amparo à Pesquisa do Estado de Minas Gerais (APQ-03239-12) e ao PET-SESU/MEC.

Todos os autores declararam não haver qualquer potencial conflito de interesses referente a este artigo.

\section{REFERÊNCIAS}

1. Yeng LT, Stump P, Kaziyama HHS, Teixeira MJ, Imamura M, Greve JMD. Medicina física e reabilitação em doentes com dor crônica. Rev Med. 2001;80:245-55

2. Herrera E, Sandoval MC, Camargo DM, Salvini TF. Motor and sensory nerve conduction are affected differently by ice pack, ice massage, and cold water immersion. Phys Ther. 2010;90(4):581-91.

3. Douris P, McKenna R, Madigan K, Cesarski B, Costiera R, Lu M. Recovery of maximal isometric grip strength following cold immersion. J Strength Cond Res. 2003;17(3):509-13.

4. Cordova ML, Bernard LW, Au KK, Demchak TJ, Stone MB, Sefton JM. Cryotherapy and ankle bracing effects on peroneus longus response during sudden inversion. J Electromyogr Kinesiol. 2010;20(2):348-53.

5. Kubo K, Kanehisa H, Fukunaga T. Effects of cold and hot water immersion on the mechanical properties of human muscle and tendon in vivo. Clin Biomech (Bristol, Avon). 2005;20(3):291-300.

6. Sanya $A O$, Bello AO. Effects of cold application on isometric strength and endurance of quadriceps femoris muscle. Afr J Med Med Sci. 1999;28(3-4):195-8.

7. Chesterton LS, Foster NE, Ross L. Skin temperature response to cryotherapy. Arch Phys Med Rehabil. 2002;83(4):543-9

8. Agne JE. Eletrotermoterapia: teoria e prática. Porto Alegre: Palotti; 2004. p. 365.

9. Alencar D, Melo TA, Matias KFS. Princípios fisiológicos do aquecimento e alongamento muscular na atividade esportiva. Rev Bras Med Esporte. 2010;16(3):230-4.

10. Weineck J. Treinamento Ideal. 9a. ed. São Paulo: Manole; 2003.

11. Edouard P, Gasq D, Calmels P, Ducrot S, Degache F. Shoulder sensorimotor control assessment by force platform: feasibility and reliability. Clin Physiol Funct Imaging. 2012;32(5):409-13.

12. Sousa FH. Uma revisão bibliográfica sobre a utilização do Nintendo ${ }^{\oplus}$ Wii como instrumento terapêutico e seus fatores de risco. REA. 2011;123(11):155-60.
13. Blackburn JT, Mynark RG, Padua DA, Guskiewicz KM. Influences of experimental factors on spinal stretch reflex latency and amplitude in the human triceps surae. J Electromyogr Kinesiol. 2006;16(1):42-50.

14. Chappell JD, Yu B, Kirkendall DT, Garrett WE. A comparison of knee kinetics between male and female recreational athletes in stop-jump tasks. Am J Sports Med. 2002;30(2):261-7.

15. Dixon PG, Kraemer WJ, Volek JS, Howard RL, Gomez AL, Comstock BA, et al. The impact of cold-water immersion on power production in the vertical jump and the benefits of a dynamic exercise warm-up. J Strength Cond Res. 2010;24(12):3313-7.

16. Roush JR, Kitamura J, Waits MC. Reference Values for the Closed Kinetic Chain Upper Extremity Stability Test (CKCUEST) for Collegiate Baseball Players. N Am J Sports Phys Ther. 2007;2(3):159-63.

17. Hamill J, Knutzen KM. Bases biomecânicas do movimento humano. 3a. ed. São Paulo: Manole; 2012.

18. Bleakley CM, Costello JT, Glasgow PD. Should athletes return to sport after applying ice? A systematic review of the effect of local cooling on functional performance. Sports Med. 2012;42(1):69-87.

19. Tremblay F, Estephan L, Legendre M, Sulpher S. Influence of local cooling on proprioceptive acuity in the quadriceps muscle. J Athl Train. 2001;36(2):119-123.

20. Rubley MD, Denegar CR, Buckley WE, Newell KM. Cryotherapy, sensation, and isometric-force variability. J Athl Train. 2003;38(2):113-119.

21. Brasileiro JS, Faria AF, Queiroz LL. Influencia do resfriamento e do aquecimento local na flexibilidade dos músculos isquiotibiais. Rev Bras Fisioter. 2007;11(1):57-61.

22. Doeringer JR, Hoch MC, Krause BA. Ice application effects on peroneus longus and tibialis anterior motoneuron excitability in subjects with functional ankle instability. Int J Neurosci. 2010;120(1):17-22.

23. Stewart D, Macaluso A, De Vito G. The effect of an active warm-up on surface EMG and muscle performance in healthy humans. Eur J Appl Physiol. 2003;89(6):509-13. 